\section{E.002 THE SWEDISH 2020 STRATEGY ON THE SAFE USE OF QUAD BIKES}

Peter Lundqvist*. Swedish University of Agricultural Sciences, Alnarp, Sweden

10.1136/injuryprev-2021-safety. 114

Context The Swedish Transport Administration together with major stakeholders including researchers from the Swedish University of Agricultural Sciences (SLU) developed a joint updated strategy for safe use of Quadbikes.

Process and analysis An evaluation of the previous strategy (2014-2020) was done regarding injury statistics, the development of safety features, education, training and research both in Sweden and at an international level. The development in Australia regarding 'Crush Protection Device (CPD)' on Quads was one of the major developments since last strategy.

Outcome The joint strategy prioritize a number of areas for further actions: a) Increased use of helmets, b) Increased use of Crush Protection Device (CPD) c) Increased use of alcohol interlocks, d) Decreased use of Quads on public roads, e) Children should not be using Quads and f) Improved information and increased education and training regarding risk awareness and safe driving.

The strategy also points out the importance of further research, development and information regarding the Crush Protection Device (CPD); alternative vehicles such as side-bysides, small tractors or off-road motorcycles; the development and use of warning/alarm systems for roll-over situations; children and Quad bikes; risks with passengers and loads as well as further education and training issues. Each of the stakeholders stated their ambitions in order to reach the joint goal to reduce the number of fatalities with $50 \%$ by the year 2030. SLU will now work with a full-scale test with Crush Protection Device (CPD) on Quad bikes, mainly during offroad use, with 100 professional users.

\section{F - Future, March 24, 2021}

\section{F.001 TRAFFIC ACCIDENTS: TECHNOLOGY THAT REBUILDS THE PAST TO SAVE THE FUTURE}

Miryam Moya*. Trimble Inc., Madrid, Spain

10.1136/injuryprev-2021-safety. 115

The accident, despite its brevity, is a dynamic process that takes place in space and time at certain points or areas and moments where events occur. The union between a point and a point takes its name from the position and involves a phase of the accident. Knowing its evolution allows investigating the applicable improvements to avoid the same errors in future cases or circumstances.

The powerful and practical solution to investigate and reconstruct traffic accidents or crime scenes, which give us the keys to avoiding them in the future, is the union of Trimble's hardware and software solution.

With the Trimble Forensics SX10 solution, forensic teams only have to pick up the instrument and work, spending less time on the scene, minimizing the danger of being exposed on a highway to collect data after a traffic accident. Parking is fast and simple, making data capture not only more efficient, but also extremely accurate. Even point cloud viewing and verification is done on site.

Once you are out of the scene of the accident and out of danger, in your office you can continue with the investigation and reconstruction of any type of traffic accident or crime scene, reaching its conclusion and being able to make the necessary decisions to avoid that Tragic events occur, in turn preventing thousands of deaths and serious injuries that will destroy the lives of millions of people around the world.

\section{F.002 COMMUNITY ENGAGEMENT FOR A SAFER FUTURE: ACTIVE TRANSPORTATION}

${ }^{1}$ Breanna Fraser-Hevlin, 2,3 Megan Oakey, ${ }^{4}$ Tobin Copley, ${ }^{1}$ Alex Zheng ${ }^{*}$ ${ }^{5}$ Ediriweera Desapriya. 'Simon Fraser University, Burnaby, Canada; ${ }^{2} B C$ Injury Research and Prevention Unit, $B C$ Children's Hospital, Vancouver, Canada; ${ }^{3} B C$ Centre for Disease Control, Vancouver, Canada; ${ }^{4}$ Fraser Health Authority, Surrey, Canada; ${ }^{5}$ University of British Columbia, Department of Pediatrics, Vancouver, Canada

\subsection{6/injuryprev-2021-safety. 116}

Background The purpose of this study is to examine the association between perception of neighborhood road safety and active modes of transportation among British Columbians to ensure healthy lives, and to make cities inclusive, safe, resilient and sustainable.

Methods A representative sample of adult (18+ years old) British Columbians was drawn from each of BC's five regional health authorities (total $\mathrm{n}=842$ ) and a survey questionnaire asking about their perceptions of neighbourhood road safety, use of physically active modes of transportation and recreation on neighbourhood streets was administered. Sample weighted values were used in all analyses reported here.

Results Among the survey participants, 65\% wanted to walk or run more in their neighbourhood and identified traffic speed and traffic volume as barriers while availability of sidewalks, street lights and crosswalks were indicated as enabling factors. $45 \%$ of survey participants wanted to cycle more in their neighbourhood and indicated speed of cars and lack of bike lanes as the discouraging factors and built environment factors, particularly bike lanes were rated as encouraging factors. Younger persons were more likely to support bike lanes whereas older persons $(50+)$ are more likely to oppose bike lanes.

Conclusion Safety perceptions of the local neighborhood road network may influence adults' use of active transportation methods.

Learning Outcomes By improving the built environment and managing traffic speed and volume, community engagement on active transportation can be enhanced to achieve the goals of safe and sustainable cities and ensure healthy lives and promote well-being for all at all ages.

\section{F.003 SPATIALLY FORECASTING SERIOUS INJURY INTO THE FUTURE}

Ben Beck*, Belinda Gabbe. Monash University, Melbourne, Australia

\subsection{6/injuryprev-2021-safety.117}

Background Understanding how injury varies across space and time is critical for informing injury prevention activities at a population level. The aim of this study was to use 